TRANSACTIONS OF THE

AMERICAN MATHEMATICAL SOCIETY

Volume 351, Number 10, Pages 4069-4088

S 0002-9947(99)02175-3

Article electronically published on July 1, 1999

\title{
INVERSE EIGENVALUE PROBLEMS ON DIRECTED GRAPHS
}

\author{
ROBERT CARLSON
}

\begin{abstract}
The differential operators $i D$ and $-D^{2}-p$ are constructed on certain finite directed weighted graphs. Two types of inverse spectral problems are considered. First, information about the graph weights and boundary conditions is extracted from the spectrum of $-D^{2}$. Second, the compactness of isospectral sets for $-D^{2}-p$ is established by computation of the residues of the zeta function.
\end{abstract}

\section{INTRODUCTION}

This work contains a number of inverse spectral results for differential operators on graphs. There are a number of physical settings where one might wish to consider differential operators on a graph: heat flow in a wire mesh, mechanical vibrations of networks of elastic strings, propagation of radiation in networks of optical fibers, and electron flow in quantum mechanical circuits. With a few recent exceptions $[9,10]$, the main mathematical studies relating operator theory and graphs are limited to difference operators $[3,4,6,15,19]$.

The point of view here is that a differential operator on a graph is like a differential operator on a one dimensional manifold. In addition to the combinatorial structure of the graph, weights are assigned to the edges to provide a metric structure. In order to have a well defined first derivative, the graph is directed. Of course the vertices are exceptional points in our topological space. Here boundary conditions are used to define the domain of the operator.

In this work we are interested in considering inverse eigenvalue problems for the second derivative and Schrödinger operators on graphs. These inverse problems have rich theories in both the classical one dimensional case and in the manifold context. Moreover, even in the one dimensional setting such basic results as compactness of isospectral classes depend on a suitable choice of boundary conditions. One of the main aims of this work is to generalize the isospectral compactness results for one dimensional periodic problems. As a consequence, we have chosen boundary conditions leading to a skew adjoint first derivative operator.

The next section contains a precise description of the operator $i D$ for certain directed graphs with weighted edges and boundary conditions at the vertices. With the appropriate domain, the operator $i D$ will be self adjoint if the conditions at the vertices are given by unitary matrices.

The third section contains inverse spectral results for the operator $-D^{2}$, which may be roughly described as follows. Typically, all eigenvalues of $-D^{2}$ are simple.

Received by the editors May 13, 1996 and, in revised form, April 7, 1997.

1991 Mathematics Subject Classification. Primary 34L05.

Key words and phrases. Inverse eigenvalue problem, graph spectral theory, zeta function.

(C)1999 American Mathematical Society 
If the eigenvalues and the edge weights are known, then the real parts of the eigenvalues of the boundary matrix may be determined. For rather trivial reasons the weights may not be determined from the eigenvalues. What may be determined typically is the sum of the edge weights for loops in the graph. These may be interpreted as the lengths of the loops, and the relationship between the eigenvalues of $-D^{2}$ and the lengths of the loops may be viewed as an analog of results relating the spectrum of the Laplacian on a manifold to the lengths of closed geodesics [7, p. 170].

The fourth section considers the zeta function $\zeta_{p}$ of $-D^{2}-p$. Within our class of operators, the singular parts of the zeta function do not depend on the boundary conditions. For the purposes of studying the residues of $\zeta_{p}$, this reduces the problem to a diagonal system of operators on circles. The standard theory then implies that isospectral classes of Schrödinger operators are compact.

Before beginning, we mention some notational conventions. The derivative with respect to $x$ is denoted $\partial_{x}$. The real and complex numbers are $\mathcal{R}$ and $\mathcal{C}$ respectively. The Hilbert space inner product is $\langle$,$\rangle .$

It a pleasure to thank M. Harmer, B. Pavlov and the referee for helpful comments.

\section{Directed Graphs AND the OPERATOR $i D$}

There is a fruitful interplay between certain graphs and differential operators. The graph $\mathcal{G}$ is assumed to be finite and directed. Multiple edges between vertices are allowed. Each vertex $v$ has $\delta(v)>0$ input (entering) edges and an equal number of output (exiting) edges. The edges, denoted $e_{n}, n=1, \ldots, N$, have weights $w_{n}>0$. In addition, each vertex $v$ has an invertible linear transformation

$$
T(v): \mathcal{C}^{\delta(v)} \rightarrow \mathcal{C}^{\delta(v)}
$$

which will be used in describing boundary conditions.

Thinking of a directed edge $e_{n}$ as an interval $\left[0, w_{n}\right]$, we consider differential operators which act by $f \rightarrow i f^{\prime}(x)$ for functions in their domain, which will be a subset of $\bigoplus_{n} L^{2}\left[0, w_{n}\right]$. While this description facilitates geometric thinking, computations are simplified by making a linear change of variables so that each edge becomes $[0,1]$. Our Hilbert space becomes the weighted space $\bigoplus_{n} L^{2}\left([0,1], w_{n}\right)$ with inner product

$$
\langle f, g\rangle=\sum_{n} \int_{0}^{1} f_{n}(x) \overline{g_{n}(x)} w_{n} d x, \quad f=\left(\begin{array}{c}
f_{1} \\
\vdots \\
f_{N}
\end{array}\right), \quad g=\left(\begin{array}{c}
g_{1} \\
\vdots \\
g_{N}
\end{array}\right) .
$$

On its domain, the operator $i D$ will act by

$$
\left(\begin{array}{c}
f_{1} \\
\vdots \\
f_{N}
\end{array}\right) \rightarrow i\left(\begin{array}{c}
\partial_{x} f_{1} / w_{1} \\
\vdots \\
\partial_{x} f_{N} / w_{N}
\end{array}\right)
$$

The local coordinate mapping of edges to intervals is chosen so that edges exit a vertex at 0 and enter at 1 . The domain of $i D$ will be determined by boundary 
conditions at the vertices. Let $F_{i}(v)$ be the $\delta(v)$ - tuple of input values of $f$ at $v$ :

$$
F_{i}(v)=\left(\begin{array}{c}
f_{n(1)}(1) \\
\vdots \\
f_{n(\delta(v))}(1)
\end{array}\right) .
$$

The $\delta(v)$-tuple of corresponding output values will be $F_{o}(v)$. An ordering of the inputs and outputs is provided by the ordering of the edges. Functions in the domain of $i D$ will be required to satisfy the vertex conditions

$$
F_{i}(v)=T(v) F_{o}(v) .
$$

Perhaps the simplest way to initiate the study of $i D$ as a Hilbert space operator is to start with the maximal operator $i D_{\max }$, whose domain consists of all $f$ : $[0,1] \rightarrow \mathcal{C}^{N}$ with absolutely continuous components and whose derivatives are in $L^{2}[0,1]$. An easy extension of standard results shows that $i D_{\max }$ is a Fredholm operator of index $N$. The operator $i D$ has domain consisting of those functions in the domain of $i D_{\max }$ which satisfy the boundary conditions (2.a). (See for instance $[12$, pp. $145,169,188,272]$.)

Let $\langle F, G\rangle_{v}$ denote the usual complex inner product on $\mathcal{C}^{\delta(v)}$. Then for functions $f, g$ in the domain of $i D$

$$
\begin{aligned}
\langle i D f, g\rangle & -\langle f, i D g\rangle=\sum_{n} \int_{0}^{1}\left[\frac{i}{w_{n}}\left(\partial_{x} f_{n}\right) \bar{g}_{n}-f_{n} \overline{\frac{i}{w_{n}} \partial_{x} g_{n}}\right] w_{n} d x \\
& =i \sum_{v}\left[\left\langle F_{i}(v), G_{i}(v)\right\rangle_{v}-\left\langle F_{o}(v), G_{o}(v)\right\rangle_{v}\right] \\
& =i \sum_{v}\left[\left\langle T(v) F_{o}(v), T(v) G_{o}(v)\right\rangle_{v}-\left\langle F_{o}(v), G_{o}(v)\right\rangle_{v}\right] \\
& =i \sum_{v}\left\langle\left(T^{*}(v) T(v)-I\right) F_{o}(v), G_{o}(v)\right\rangle_{v} .
\end{aligned}
$$

Here $T^{*}(v)$ denotes the conjugate transpose of $T(v)$.

Since the domain of $i D$ is defined by $N$ boundary conditions, $i D$ is Fredholm with index at least 0 . Suppose that $T^{*}(v) T(v)-I=0$ for all vertices $v$. The computation (2.b) shows that $i D$ has no eigenvalues with nonzero imaginary part, so the index is 0 and $i D$ is self adjoint. Henceforth the matrices $T(v)$ are assumed to be unitary.

This discussion is summarized in the following result.

Proposition 2.1. Suppose that the matrix $T(v)$ is unitary for each vertex $v \in \mathcal{G}$. Let $i D$ be the operator

$$
\left(\begin{array}{c}
f_{1} \\
\vdots \\
f_{N}
\end{array}\right) \rightarrow i\left(\begin{array}{c}
\partial_{x} f_{1} / w_{1} \\
\vdots \\
\partial_{x} f_{N} / w_{N}
\end{array}\right)
$$

on $\bigoplus_{n} L^{2}\left([0,1], w_{n}\right)$, whose domain consists of all $f:[0,1] \rightarrow \mathcal{C}^{N}$ whose components are absolutely continuous with derivatives in $L^{2}[0,1]$ and which satisfy the boundary conditions (2.a). Then $i D$ is self adjoint. 
It will be convenient to let $W$ denote the diagonal matrix $\operatorname{diag}\left[w_{n}\right]$. Any eigenfunction for $\pm i D$ must satisfy the system of equations

$$
\pm i W^{-1} \partial_{x} Y=\lambda Y, \quad Y(x, \lambda)=\left(\begin{array}{c}
y_{1}(x, \lambda) \\
\vdots \\
y_{N}(x, \lambda)
\end{array}\right) .
$$

The solution matrix

$$
\mathbf{Y}=\exp (\mp i W \lambda x)
$$

has columns forming a basis of solutions to (2.c). The conditions (2.a) defining the domain of $i D$ may be written as a single linear system

$$
f_{m}(1)-\sum_{n=1}^{N} T_{m n} f_{n}(0)=0, \quad m=1, \ldots, N,
$$

and $\lambda$ will be an eigenvalue of $\pm i D$ exactly when the condition

$$
\operatorname{det}[\mathbf{Y}(1, \lambda)-T \mathbf{Y}(0, \lambda)]=\operatorname{det}[\exp (\mp i W \lambda)-T]=0, \quad T=\left(T_{m n}\right),
$$

is satisfied.

An explicit description of the resolvent

$$
R_{1}(\lambda)=[i D-\lambda I]^{-1}
$$

will be useful. Solutions of

$$
i W^{-1} \partial_{x} Y-\lambda Y=f, \quad Y=\left(\begin{array}{c}
y_{1} \\
\vdots \\
y_{N}
\end{array}\right), \quad f=\left(\begin{array}{c}
f_{1} \\
\vdots \\
f_{N}
\end{array}\right)
$$

have the form

$$
Y(x, \lambda)=\exp (-i \lambda x W) K(\lambda)-i \int_{0}^{x} \exp (i \lambda[t-x] W) W f(t) d t, \quad K(\lambda) \in C^{N} .
$$

Satisfaction of the boundary conditions $T Y(0)-Y(1)=0$ requires

$$
K(\lambda)=i[\exp (-i \lambda W)-T]^{-1} \int_{0}^{1} \exp (i \lambda[t-1] W) W f(t) d t
$$

For notational convenience let

$$
M(\lambda)=[I-\exp (i \lambda W) T]^{-1} .
$$

Thus

$$
\begin{aligned}
{\left[R_{1}(\lambda) f\right](x) } & =i \exp (-i \lambda x W) M(\lambda) \int_{0}^{1} \exp (i \lambda t W) W f(t) d t \\
& -i \int_{0}^{x} \exp (i \lambda[t-x] W) W f(t) d t
\end{aligned}
$$




\section{SPECTRAL THEORY FOR $-D^{2}$}

This section contains an analysis of the generic structure of the spectrum for $i D$ and $-D^{2}$. Constructive procedures are presented for recovering information about the graph $\mathcal{G}$ from the eigenvalues of $-D^{2}=[i D]^{2}$. The information to be obtained consists of the real parts of the eigenvalues of the boundary matrix $T$ and the lengths of loops in $\mathcal{G}$. Our initial focus is on the function

$$
P(\lambda)=\operatorname{det}[\exp (-i W \lambda)-T]
$$

whose zeroes are the eigenvalues of $i D$.

3.1. Generic behaviour. The justification of the inverse spectral procedures makes use of a number of assumptions about the operator $-D^{2}$. These assumptions include the following:

(i) each eigenvalue $\lambda_{k}$ of $i D$ has a corresponding eigenspace of dimension 1 ,

(ii) each eigenvalue $\lambda_{k}$ of $i D$ is a simple root of $P(\lambda)$.

For some results, arithmetic conditions on the weights $w_{n}$ will be assumed. The various assumptions about the operator $-D^{2}$ are generically valid in senses which will be made precise.

For the first result the connectivity of $\mathcal{G}$ is ignored.

Proposition 3.1. For every set of weights $\left\{w_{n}\right\}$ there is a unitary matrix $T$ whose associated operator iD satisfies conditions (i) and (ii).

Proof. The examples are found in the trivial case when our system is completely decoupled, the matrix $T$ having the form

$$
T=\operatorname{diag}\left[\exp \left(i \theta_{n}\right)\right]
$$

In this case eigenvalues of $i D$ have the form

$$
\theta_{n} / w_{n}+2 \pi k / w_{n}, \quad n=1, \ldots, N, \quad k=0, \pm 1, \pm 2, \ldots
$$

Condition $(i)$ will be satisfied unless

$$
\theta_{m} / w_{m}+2 \pi j / w_{m}=\theta_{n} / w_{n}+2 \pi k / w_{n}
$$

or

$$
\theta_{m}=\frac{w_{m}}{w_{n}}\left[\theta_{n}+2 \pi k\right] \bmod 2 \pi .
$$

The values of $\theta_{m}$ may be chosen inductively. The first value $\theta_{1} \in[0,2 \pi)$ is arbitrary. Subsequent choices are only excluded from a countable set of values, which shows that $(i)$ may be satisfied.

If $T$ has the specified form,

$$
P(\lambda)=\prod_{n=1}^{N}\left[\exp \left(i w_{n} \lambda\right)-\exp \left(i \theta_{n}\right)\right] .
$$

If $\sigma_{m, k}$ is one of the eigenvalues $\lambda_{j}$ of the form

$$
\sigma_{m, k}=\theta_{m} / w_{m}+2 \pi k / w_{m}, \quad m=1, \ldots, N, \quad k=0, \pm 1, \pm 2, \ldots,
$$

then

$$
\partial_{\lambda} P\left(\sigma_{m, k}\right)=i w_{m} \exp \left(i w_{n} \sigma_{m, k}\right) \prod_{n \neq m}\left[\exp \left(i w_{n} \sigma_{m, k}\right)-\exp \left(i \theta_{n}\right)\right] \neq 0 .
$$

It will also be important to observe that Proposition 3.1 can be satisfied with matrices $T(v)$ respecting the connectivity of $\mathcal{G}$. 
Proposition 3.2. For every set of weights $\left\{w_{n}\right\}$ there is a collection of unitary matrices

$$
T_{0}(v): \mathcal{C}^{\delta(v)} \rightarrow \mathcal{C}^{\delta(v)}
$$

whose associated operator iD satisfies conditions (i) and (ii).

Proof. This more restrictive case may be reduced to the previous one.

Start at any vertex and follow a directed edge path until a vertex $v$ is repeated. Since each vertex of $\mathcal{G}$ has the same number of input and output edges, the edges of the path from $v$ to $v$ may be removed from $\mathcal{G}$, leaving another graph of the same type. Thus $\mathcal{G}$ may be decomposed into an edge disjoint collection of closed paths.

Let $\gamma$ be one of these closed paths, and select one vertex $v$ from $\gamma$. At each of the other vertices $w$ of $\gamma$ there is one entering edge $e_{i}$ and one exiting edge $e_{j}$. Let $T$ be partially defined by the condition $f_{i}(1)=f_{j}(0)$, that is

$$
T_{i j}=1, \quad T_{l j}=0, \quad l \neq i .
$$

These closed paths may now be considered intervals with weights $\sum w_{m}$, the sum taken over the edges in the path. The proof of Proposition 3.1 implies that values $\exp \left(i \theta_{v}\right)$ may be selected for boundary conditions at $v$ giving the eigenvalue conditions $(i)$ and $(i i)$ as before.

Propositions 3.1 and 3.2 can be extended to generic operators of the form $i D$ by using some results on analytic families of operators. Let $T_{0}$ and $T_{1}$ be unitary matrices with logarithms $i A_{0}$ and $i A_{1}$ :

$$
T_{j}=\exp \left(i A_{j}\right), \quad A_{j}=A_{j}^{*}, \quad j=0,1 .
$$

The real analytic path of unitary matrices

$$
T(s)=\exp \left(i\left[(1-s) A_{0}+s A_{1}\right]\right), \quad-\infty<s<\infty,
$$

satisfies $T(0)=T_{0}$ and $T(1)=T_{1}$. Denote by $i D_{s}$ the operator $i D$ with domain defined by the boundary conditions using the unitary matrix $T(s)$. The corresponding resolvents are denoted $R_{s}(\lambda)$.

Lemma 3.3. The eigenvalues $\lambda_{k}(s)$, appearing with the appropriate multiplicity, are well defined real analytic functions of $s$. There is also a corresponding complete orthonormal real analytic family $\left\{\psi_{k}(s)\right\}$ of eigenvectors for $i D_{s}$.

Proof. From the explicit formula (2.e) we see that if $s \in(a, b)$ and $\lambda$ is real and in the resolvent set of $i D_{s}$, then the operators $R_{s}(\lambda)$ form a self adjoint holomorphic family [12, p. 385] of compact operators. The eigenvalues $\lambda_{k}(s)$ of $i D_{s}$ and the eigenvalues $\nu_{k}(s)$ of $R_{s}(\lambda)$ are related by

$$
\nu_{k}(s)=\frac{1}{\lambda_{k}(s)-\lambda} .
$$

For each $s_{0} \in \mathcal{R}$ there are a real $\lambda$ in the resolvent set $\rho\left(i D_{s_{0}}\right)$ of $i D_{s_{0}}$ and an interval $(a, b)$ containing $s_{0}$ such that for all $s \in(a, b)$ we have $\lambda \in \rho\left(i D_{s}\right)$. Since the operators $R_{s}(\lambda)$ are resolvents, 0 is never an eigenvalue of $R_{s}(\lambda)$. The discussion in $\left[12\right.$, p. 393] shows that each $\nu_{k}(s)$ is a real analytic function for $s \in(a, b)$. By (3.a) the same is true for $\lambda_{k}(s)$.

If $t_{0}, t_{1} \in \mathcal{R}$ there is a finite open cover of $\left[t_{0}, t_{1}\right]$ by intervals $\left(a_{j}, b_{j}\right)$ such that $\left(a_{j}, b_{j}\right) \cap\left(a_{j+1}, b_{j+1}\right) \neq \emptyset$ and such that the eigenvalues of $i D_{s}$ are real analytic in $\left(a_{j}, b_{j}\right)$. Now index the eigenvalues $\lambda_{k}(s)$ according to their index in the first 
interval $\left(a_{1}, b_{1}\right)$ and extend these functions from $\left(a_{j}, b_{j}\right)$ to $\left(a_{j+1}, b_{j+1}\right)$ by requiring the definition to be consistent on $\left(a_{j}, b_{j}\right) \cap\left(a_{j+1}, b_{j+1}\right)$.

The eigenvectors are handled analogously.

Suppose that $\mathcal{G}$ has $M$ vertices. The $M$-tuple of transition matrices defining the domain of $i D$ is an element of the product of unitary groups $\mathcal{U}=\chi_{m=1}^{M} U\left(\delta\left(v_{m}\right)\right)$. The next result considers the size of the set in this product where conditions $(i)$ and $(i i)$ do not hold.

Theorem 3.4. Suppose that the graph $\mathcal{G}$ has arbitrary weights $\left\{w_{n}\right\}$ with in and out degrees $\delta(v)$ at the vertices $v$. Then conditions (i) and (ii) hold except for a set of transition matrices having measure zero in $\mathcal{U}=\chi_{m=1}^{M} U\left(\delta\left(v_{m}\right)\right)$.

Proof. The proof begins by showing that conditions $(i)$ and $(i i)$ hold at most points along certain curves in $\mathcal{U}$. According to Proposition 3.2 there is some choice of unitary transition matrices

$$
T_{0}(v): \mathcal{C}^{\delta(v)} \rightarrow \mathcal{C}^{\delta(v)}
$$

satisfying conditions $(i)$ and $(i i)$. There are self adjoint matrices $A_{0}(v)$ and $A(v)$ such that

$$
T_{0}(v)=\exp \left(i A_{0}(v)\right), \quad T(v)=\exp (i A(v)) .
$$

Define $T_{s}(v)=\exp \left(i\left[(1-s) A_{0}(v)+s A(v)\right]\right)$.

By Lemma 3.3 the eigenvalues (with multiplicity) and orthonormal eigenvectors of $i D_{s}$ may be chosen to be real analytic functions of $s$. Now condition $(i)$ fails if and only if $\lambda_{j}(s)-\lambda_{k}(s)=0$ for some $j \neq k$. Since none of these differences is 0 for all $s$, each may vanish only finitely many times between $s=0$ and $s=1$. Thus there are at most countably many points $s$ where $(i)$ does not hold.

A similar argument works for condition $(i i)$. The function $\partial_{\lambda} P\left(\lambda_{j}(s)\right)$ is analytic in $s$. At $s=0$ we have $\partial_{\lambda} P\left(\lambda_{j}(0)\right) \neq 0$, so that $\partial_{\lambda} P\left(\lambda_{j}(s)\right) \neq 0$ for all $j$ except at countably many values of $s$.

The remainder of the proof consists of extending the result obtained so far to a measure theoretic statement on $\mathcal{U}$. Only a sketch of the argument is provided.

First note that the self adjoint $\delta\left(v_{m}\right) \times \delta\left(v_{m}\right)$ matrices may be considered a complex Euclidean space by ignoring the entries below the diagonal. The exponential map $A \rightarrow \exp (i A)$ is a local diffeomorphism from the self adjoint matrices onto $U\left(\delta\left(v_{m}\right)\right)$. Let $K$ be a positive integer. It is possible to find a finite open covering $\left\{V_{\alpha}\right\}$ and numbers $r_{\alpha}$ with $K<r_{\alpha}<K+1$ such that $\pm r_{\alpha}$ are in the resolvent set for all operators $i D$ defined by transition matrices in $V_{\alpha}$. Since the set of transition matrices $\{T(v)\}$ for which $(i)$ and $(i i)$ hold is dense, we may take $V_{\alpha}$ to be the image, under the exponential map, of a Euclidean ball, and the center point of the ball gives an operator for which $(i)$ and $(i i)$ hold.

Using the ideas in the proof of Lemma 3.3, and in [12, pp. 109, 116], the eigenvalues $\lambda_{1}, \ldots, \lambda_{k}$ with absolute value smaller than $r_{\alpha}$ are continuous functions in $V_{\alpha}$. Two of these eigenvalues agree when $\prod_{i \neq j}\left(\lambda_{i}-\lambda_{j}\right)=0$, and this zero set $E(\alpha, K)$ is measurable in $V_{\alpha}$. To get the measure of this set, integrate its characteristic function in polar coordinates. By the first part of the proof the integral is zero along each radial line, so the set $E(\alpha, K)$ has measure 0 . Finally, the set of unitary matrices for which condition $(i)$ fails is $\bigcup_{K} \bigcup_{\alpha} E(\alpha, K)$, which has measure 0 .

Again a similar argument works for condition $(i i)$. 
Having considered the generic behaviour of $i D$ for $\mathcal{T}=\left(T\left(v_{1}\right), \ldots, T\left(v_{M}\right)\right) \in \mathcal{U}$, we now derive a basic result for the general case.

Theorem 3.5. Suppose that $\lambda_{k}(\mathcal{T})$ are the eigenvalues of $i D$, listed with multiplicity given by the dimension of the corresponding eigenspace. Let $m$ be the dimension of the null space of $i D$. Then there are complex numbers $\beta$ and $C$, with $C \neq 0$, such that

$$
P(\lambda)=C \lambda^{m} e^{\beta \lambda} \prod_{k=1}^{\infty}\left(1-\lambda / \lambda_{k}\right) e^{\lambda / \lambda_{k}} .
$$

In particular, the multiplicities given by the dimension of the eigenspaces agrees with the analytic multiplicities of $\lambda_{k}$ as a root of $P(\lambda)$.

Proof. It will be necessary to have a coarse estimate for the distribution of eigenvalues for $i D$. If the boundary conditions defining the domain of $i D$ were $f_{n}(0)=f_{n}(1)$ for $n=1, \ldots, N$, then a direct computation would show that the eigenvalues are $2 \pi k / w_{n}$ for integer $k$ and $n=1, \ldots, N$. Since the examples of interest differ from this trivial one only in the set of $N$ boundary conditions, it is elementary ([2], Lemma 1.2) to show that the number of eigenvalues appearing in any open interval, counting with multiplicity, cannot change by more than $N$.

For notational simplicity assume that $m=0$. The entire function $P(\lambda)$ has order 1, so by Hadamard's theorem [1, p. 207] its genus is either 0 or 1 . Since $\sum 1 /\left|\lambda_{k}\right|=\infty$ and $\sum 1 /\left|\lambda_{k}\right|^{2}<\infty$, the genus [1, p. 195] is 1 and $P(\lambda)$ has a product representation

$$
P(\lambda)=C e^{\beta \lambda} \prod_{k=1}^{\infty}\left(1-\lambda / \sigma_{k}\right) e^{\lambda / \sigma_{k}} .
$$

In this representation the set of numbers $\left\{\sigma_{k}\right\}$ is the same as the set $\left\{\lambda_{k}\right\}$, but the multiplicities might be different. According to Theorem 3.4 these multiplicities agree for a dense set in $\mathcal{U}$. We will write $\mathcal{T} \in \mathcal{U}$ as the limit of a sequence $\mathcal{T}_{n} \in \mathcal{U}$ satisfying $(i)$ and $(i i)$, and use a continuity argument to show that the multiplicities must agree for all $\mathcal{T}$.

From the definition

$$
P(\lambda, \mathcal{T})=\operatorname{det}[\exp (-i W \lambda)-T]
$$

it is evident that $P\left(\lambda, \mathcal{T}_{n}\right) \rightarrow P(\lambda, \mathcal{T})$ uniformly on compact subsets of $\mathcal{C}$. It is a consequence of Rouche's theorem [1, p. 152] that each root $\sigma$ of $P(\lambda)$ of multiplicity $M$ is the limit of exactly $M$ eigenvalues $\lambda_{k}\left(\mathcal{T}_{n}\right)$.

On the other hand, (2.e) shows that the resolvents for $i D$ corresponding to $\mathcal{T}_{n}$ converge uniformly in operator norm to the resolvent corresponding to $\mathcal{T}$ on compact subsets of the resolvent set corresponding to $\mathcal{T}$. Suppose that $\lambda(\mathcal{T})$ is an eigenvalue of $i D$ with eigenspace of dimension $M$. Then since $i D$ is self adjoint, the eigenprojection [12, p. 181] associated to $\lambda(\mathcal{T})$ has rank $M$ and [12, pp. 213-214] is the limit of exactly $M$ eigenvalues $\lambda_{k}\left(\mathcal{T}_{n}\right)$.

From the observations of the previous two paragraphs, we see that the multiplicities given by the dimension of the eigenspaces agree with the analytic multiplicities of $\lambda_{k}$ as a root of $P(\lambda)$. Consequently, we may take $\sigma_{k}=\lambda_{k}$ in the formula for $P(\lambda)$. 
3.2. Inverse problems. Now we consider recovering graph information from the eigenvalues $\mu_{k}$ of operators $-D^{2}$. In the general case it will be necessary to assume that the multiplicities of the eigenvalues, that is the dimensions of the eigenspaces, are also known. However Theorem 3.4 shows that the generic case has all eigenvalues simple.

Of course having the values $\mu_{k}$ is the same as knowing the magnitudes $\left|\lambda_{k}\right|$ of the eigenvalues for $i D$. Although the signs of the eigenvalues are unknown, we do know that the set of values $\pm\left|\lambda_{k}\right|$ comprises the union of the spectra of $\pm i D$. By (2.d) these values are the roots of $\operatorname{det}[\exp (-i W \lambda)-T]=0$ and $\operatorname{det}[\exp (i W \lambda)-T]=0$. Since

$$
\operatorname{det}[\exp (i W \lambda)-T]=\operatorname{det}[T] \operatorname{det}\left[T^{-1}-\exp (-i W \lambda)\right] \operatorname{det}[\exp (i W \lambda)],
$$

this second equation may be replaced with the equivalent condition

$$
\operatorname{det}\left[T^{-1}-\exp (-i W \lambda)\right]=0 .
$$

Thus we consider the entire function

$$
\begin{gathered}
Q(\lambda)=\operatorname{det}[\exp (-i W \lambda)-T]\left[T^{-1}-\exp (-i W \lambda)\right] \\
=-\operatorname{det}\left[I-T \exp (-i W \lambda)-\exp (-i W \lambda) T^{-1}+\exp (-2 i W \lambda)\right] .
\end{gathered}
$$

Lemma 3.6. The eigenvalues $\mu_{k}$ of $-D^{2}$ together with their multiplicities determine the function

$$
Q(\lambda)=\operatorname{det}[\exp (-i W \lambda)-T]\left[T^{-1}-\exp (-i W \lambda)\right] .
$$

Proof. By Theorem 3.5 and (3.b), $Q(\lambda)$ has the product representation

$$
Q(\lambda)=C \lambda^{m} e^{\alpha \lambda}\left[\prod_{k=1}^{\infty}\left(1-\lambda /\left|\lambda_{k}\right|\right) e^{\lambda /\left|\lambda_{k}\right|}\right]\left[\prod_{k=1}^{\infty}\left(1+\lambda /\left|\lambda_{k}\right|\right) e^{-\lambda /\left|\lambda_{k}\right|}\right], \quad\left|\lambda_{k}\right| \neq 0 .
$$

Let $Q_{1}(\lambda)$ be the entire function

$$
Q_{1}(\lambda)=\lambda^{m}\left[\prod_{k=1}^{\infty}\left(1-\lambda /\left|\lambda_{k}\right|\right) e^{\lambda /\left|\lambda_{k}\right|}\right]\left[\prod_{k=1}^{\infty}\left(1+\lambda /\left|\lambda_{k}\right|\right) e^{-\lambda /\left|\lambda_{k}\right|}\right], \quad\left|\lambda_{k}\right| \neq 0
$$

constructed from the values $\mu_{k}$.

Restrict $\lambda$ to the imaginary axis, $\lambda=i \sigma$. From the form of $Q(\lambda)$ it follows that

$$
\lim _{\sigma \rightarrow-\infty} Q(i \sigma)=-1
$$

From the product representation (3.c) and the limiting behaviour (3.d) it follows that there is a value of $\alpha$ such that

$$
\lim _{\sigma \rightarrow-\infty} e^{i \alpha \sigma} Q_{1}(i \sigma)
$$

has a nonzero complex value. It is easily seen that this choice of $\alpha$ is unique, so that the condition (3.d) determines $\alpha$, and then $C$.

Consider the case when the weights have a known common value $w_{n}=w$. Then Lemma 3.6 says we know the function

$$
\begin{aligned}
& q(\lambda)=-\operatorname{det}\left[I-T \exp (-i w \lambda)-\exp (-i w \lambda) T^{-1}+\exp (-2 i w \lambda) I\right] \\
= & -\exp (-2 i w N \lambda) \operatorname{det}\left[\exp (2 i w \lambda) I-\exp (i w \lambda) T-T^{-1} \exp (i w \lambda)+I\right] .
\end{aligned}
$$


In this case $2 N w$ can be determined by the asymptotics as $\lambda \rightarrow i \infty$ and so $N$ is determined. Construct

$$
-\exp (i N w \lambda) q(\lambda) / 2^{N}=\operatorname{det}[\cos (w \lambda) I-\operatorname{Re}(T)] .
$$

Let the eigenvalues of $T$ be $\phi_{1}, \ldots, \phi_{N}$. By taking $\lambda=\cos ^{-1}(x) / w$ for $-1 \leq x \leq 1$ we obtain the characteristic polynomial

$$
\operatorname{det}[x I-\operatorname{Re}(T)]=\left(x-\operatorname{Re}\left(\phi_{1}\right)\right) \cdots\left(x-\operatorname{Re}\left(\phi_{N}\right)\right) .
$$

This establishes the following result.

Theorem 3.7. If the weights have a known common value $w_{n}=w$, then the eigenvalues $\mu_{k}$ of $-D^{2}$ together with their multiplicities determine $N$ and the real parts of the eigenvalues $\phi_{n}$ of the unitary matrix $T$.

The case of known distinct weights can be reduced to the previous case if a mild arithmetic condition [13, pp. 37-40] is satisfied.

Theorem 3.8. Suppose that the eigenvalues $\mu_{k}$ of $-D^{2}$ together with their multiplicities are known. If in addition the weights $w_{n}$ are given and the line

$$
\lambda\left(w_{1}, \ldots, w_{N}\right) \quad \bmod 2 \pi Z^{N}
$$

is dense in the torus $R^{N} /\left[2 \pi Z^{N}\right]$, then the real parts of the eigenvalues $\phi_{n}$ of the unitary matrix $T$ may be determined.

Proof. Since the weights are known, Lemma 3.6 implies that the function

$$
\begin{array}{r}
q_{1}(\lambda)=-2^{-N} \operatorname{det}[\exp (i W \lambda / 2)] Q(\lambda) \operatorname{det}[\exp (i W \lambda / 2)] \\
=\operatorname{det}[\cos (\lambda W) I-\exp (i W \lambda / 2) T \exp (-i W \lambda / 2) / 2 \\
\left.-\exp (-i W \lambda / 2) T^{-1} \exp (i W \lambda / 2) / 2\right]
\end{array}
$$

can be constructed. Pick a number $x \in[-1,1]$ and choose a sequence of points $\lambda_{r}$ such that

$$
\lambda_{r}\left(w_{1}, \ldots, w_{N}\right) \rightarrow\left(\cos ^{-1}(x), \ldots, \cos ^{-1}(x)\right) \bmod 2 \pi Z^{N}
$$

Then

$$
q_{1}\left(\lambda_{r}\right) \rightarrow \operatorname{det}[x I-\operatorname{Re}(T)]
$$

Since $x$ was arbitrary we again recover the characteristic polynomial of $\operatorname{Re}(T)$.

Finally we consider extracting information about the weights from the eigenvalues $\mu_{k}$. This analysis begins with the forward problem of describing the function $P(\lambda)=\operatorname{det}[\exp (-i W \lambda)-T]$, assuming that we know the weights $w_{n}$. Recall that the matrix $T$ has entries $T_{i j}$ which can only be nonzero when $i$ and $j$ are respectively the indices of an input edge and an output edge at some vertex $v$. Following the terminology of topology rather than graph theory, define a cycle to be the sum of closed (directed) paths [1, p. 138].

Lemma 3.9. Suppose that the diagonal terms $T_{i i}$ are all zero. Then $P(\lambda)$ is a linear combination of exponentials

$$
P(\lambda)=\operatorname{det}[\exp (-i W \lambda)-T]=\sum_{k} c_{k} \exp \left(-i \lambda \sum_{n=1}^{N(k)} w_{n}\right)
$$

where each nonzero sum of weights is taken over a cycle of at most $N$ edges. 
Proof. Let $a_{i j}(\lambda)$ denote the entries of the matrix $\exp (-i W \lambda)-T$. The function $P(\lambda)$ may be written as a sum of products, each summand having the form $S_{\sigma}(\lambda)=$ $\operatorname{sgn}(\sigma) \prod a_{i \sigma(i)}$ where $\sigma$ is a permutation of the indices $1, \ldots, N$.

Select a vertex $v$ with $\delta(v)$ input edges $m_{1}, \ldots, m_{\delta}$ and output edges $n_{1}, \ldots, n_{\delta}$. The condition $T_{i i}=0$ means that no input edge index at $v$ is also an output edge index at $v$. Let $S_{\sigma}(\lambda)$ be a nonzero summand whose permutation $\sigma$ fixes exactly $r$ of the input edge indices at $v$, i.e. $\sigma\left(m_{k}\right)=m_{k}$. Then $S_{\sigma}(\lambda)$ has the $r$ factors $\exp \left(-i w_{m_{k}} \lambda\right)$.

Next we consider the columns whose indices $n_{l}$ are output edge indices at $v$. Since $T_{i n_{l}}=0$ unless $i \in\left\{m_{1}, \ldots, m_{\delta}\right\}$, and the $r$ row indices $m_{k}$ are already accounted for, there can be at most $\delta(v)-r$ factors $a_{i n_{l}}$ in $S_{\sigma}$ with $i \in\left\{m_{1}, \ldots, m_{\delta}\right\}$. The only other nonzero entries $a_{i j}$ are on the diagonal, so that for at least $r$ values of the output edge indices $n_{l}, \sigma\left(n_{l}\right)=n_{l}$.

The roles of input and output edges are interchangeable, so each product has an equal number of distinct diagonal contributions from inputs and outputs at each vertex. Of course each output edge at $v$ is the input at another vertex. Decomposing $\mathcal{G}$ into edge disjoint loops as in the proof of Proposition 3.2 gives the result.

Rather than $P(\lambda)$, it is the function $Q(\lambda)$ which can be determined from the eigenvalues $\mu_{k}$ by Lemma 3.6. Recall that $\mathcal{G}$ has $M$ vertices and $\mathcal{U}=\chi_{m=1}^{M} U\left(\delta\left(v_{m}\right)\right)$.

Theorem 3.10. Suppose that the diagonal terms $T_{i i}$ are all zero. Then $Q(\lambda)$ is a linear combination of exponentials

$$
Q(\lambda)=\operatorname{det}[\exp (-i W \lambda)-T]\left[T^{-1}-\exp (-i W \lambda)\right]=\sum_{k} c_{k} \exp \left(-i \lambda \sum_{n=1}^{N(k)} w_{n}\right)
$$

where each nonzero sum of weights is taken over a cycle of at most $2 N$ edges. For an open dense set of unitary matrices $\left(T\left(v_{1}\right), \ldots, T\left(v_{M}\right)\right) \in \mathcal{U}$, each frequency which is the length of a loop in $\mathcal{G}$ with no repeated edges appears with a nonzero coefficient $c_{k}$.

Proof. Since $T^{-1}=T^{*}$ Lemma 3.9 applies to both factors $\operatorname{det}[\exp (-i W \lambda)-T]$ and $\operatorname{det}\left[T^{-1}-\exp (-i W \lambda)\right]$ of $Q(\lambda)$. This shows immediately that $Q(\lambda)$ is the desired linear combination of exponentials.

To describe the generic behaviour we first consider some special examples. Partition $\mathcal{G}$ into edge disjoint loops with no repeated edges. If $v$ is a vertex in one of the loops, with input edge $i$ and output edge $j$, let $T$ be defined by requiring $f_{i}(1)=f_{j}(0)$, except at the initial vertex where the condition will be $f_{i}(1)=-f_{j}(0)$. In this case we may reindex the edges so that $P(\lambda)$ is the determinant of a block diagonal matrix with blocks of the form

$$
\left(\begin{array}{cccccc}
\exp \left(-i w_{1} \lambda\right) & -1 & 0 & 0 & \ldots & 0 \\
0 & \exp \left(-i w_{2} \lambda\right) & -1 & 0 & \ldots & 0 \\
0 & 0 & \vdots & \vdots & \ldots & 0 \\
0 & 0 & 0 & \ldots & \exp \left(-i w_{m-1} \lambda\right) & -1 \\
1 & 0 & 0 & 0 & \ldots & \exp \left(-i w_{m} \lambda\right)
\end{array}\right)
$$

Thus after the reindexing $P(\lambda)$ is a product of terms of the form

$$
\exp \left(-i \lambda \sum w_{n}\right)+(-1)^{m-1}(-1)^{m-1}=\exp \left(-i \lambda \sum w_{n}\right)+1 .
$$


The second factor $\operatorname{det}\left[T^{-1}-\exp (-i W \lambda)\right]$ of $Q(\lambda)$ is, except for a factor -1 , a product of terms of the same form. For this example each frequency which is the length of a loop in the partition of $\mathcal{G}$ with no repeated edges appears with a nonzero coefficient.

In the general case the coefficient $c_{k}$ of a term $\exp \left(-i \lambda \sum_{n} w_{n}\right)$ with frequency $\sum_{n} w_{n}$ is a polynomial in the entries of the matrices $T\left(v_{m}\right)$ and $T^{*}\left(v_{m}\right)$. As in Theorem 3.4, local coordinates for $U(\delta(v))$ may be chosen by using the exponential map $A \rightarrow \exp (i A)$ where $A=A^{*}$. In these coordinates the real and imaginary parts of the coefficient $c_{k}$ are real analytic functions of the real and imaginary parts of the entries of the matrices $A$. Our examples show that each loop length with no repeating edges has an associated nonzero coefficient $c_{k}$ for some choice of $\left(T\left(v_{1}\right), \ldots, T\left(v_{M}\right)\right)$. By the real analyticity, this particular coefficient must be nonzero on an open dense set in $\mathcal{U}$. But then all coefficients coming from lengths of loops without repeating edges are nonzero on the intersection of finitely many open dense sets, which completes the proof.

If $\lambda$ is restricted to real values, $Q(\lambda)$ is almost periodic. The distinct frequencies $\sum_{n=1}^{N(k)} w_{n}$ and the corresponding nonzero coefficients may be identified by considering the integrals [13, p. 14]

$$
\frac{1}{2 T} \int_{-T}^{T} Q(\lambda) \exp (i \nu \lambda) d \lambda
$$

\section{The Zeta Function for Schrödinger's Operator}

In this section attention shifts from $-D^{2}$ to the operator $-D^{2}-p$, where $p$ is a real valued function on $\mathcal{G}$ with several continuous derivatives. This operator has a zeta function $\zeta_{p}(s)$ much as in the smooth manifold case [18]. In the graph setting the resolvent of $-D^{2}$ can be exactly computed. Up to negligible corrections, all of the boundary conditions we have considered lead to the same diagonal for the kernel of the resolvent. This observation means that the singular part of $\zeta_{p}(s)$ can be computed by considering boundary conditions for which the graph reduces to edge disjoint loops. The local formulas for the residues of $\zeta_{p}(s)$ in such a periodic case have been known for a long time [8]. A well-known argument [14, p. 226] shows that the isospectral classes are compact.

4.1. The resolvent of $-D^{2}$. The goal for this section is to establish the following description of the resolvent of $-D^{2}$. Recall that $M(\lambda)=[I-\exp (i \lambda W) T]^{-1}$. For notational convenience, let $\lambda=-\omega^{2}$.

Lemma 4.1. The resolvent $R(\lambda)$ of $-D^{2}$ is an integral operator satisfying

$$
[R(\lambda) f](x)
$$

$$
\begin{gathered}
=-\frac{1}{2 \omega} \int_{0}^{1} \exp (-x \omega W) M(-i \omega)[\exp (2 \omega W)-I] M(i \omega) \exp (-\omega t W) W f(t) d t \\
+\frac{1}{2 \omega} \int_{0}^{1} \exp (-x \omega W) M(-i \omega)[\exp (2 \omega W)-\exp (2 t \omega W)] \exp (-\omega t W) W f(t) d t \\
\quad+\frac{1}{2 \omega} \int_{0}^{1} \exp (-\omega x W)[\exp (2 x \omega W)-I] M(i \omega) \exp (-\omega t W) W f(t) d t \\
-\frac{1}{2 \omega} \int_{0}^{x} \exp (-\omega x W)[\exp (2 x \omega W)-\exp (2 t \omega W)] \exp (-t \omega W) W f(t) d t
\end{gathered}
$$


For all $j, k=0,1,2, \ldots$ and $\epsilon>0$ the diagonal of the resolvent kernel has the following behaviour as $\omega \rightarrow \infty$ :

$$
\partial_{x}^{j} \partial_{\lambda}^{k} R(x, x, \lambda)=\partial_{x}^{j} \partial_{\lambda}^{k} \frac{1}{2 \omega} W+O\left(\exp \left(-\min _{n}\left[w_{n} / 2-\epsilon\right] \omega\right)\right) .
$$

Proof. Using (2.e) and

$$
R\left(-\omega^{2}\right)=\left[-D^{2}+\omega^{2}\right]^{-1}=(i D+i \omega)^{-1}(i D-i \omega)^{-1}
$$

leads to

$$
\begin{gathered}
{[R(\lambda) f](x)=\left[R_{1}(-i \omega) R_{1}(i \omega) f\right](x)} \\
=\exp (-x \omega W) M(-i \omega) \int_{0}^{1} i \exp (s \omega W) W \\
\times\left[\exp (s \omega W) M(i \omega) \int_{0}^{1} i \exp (-t \omega W) W f(t) d t-\int_{0}^{s} i \exp (-[t-s] \omega W) W f(t) d t\right] d s \\
-\int_{0}^{x} i \exp ([s-x] \omega W) W \\
\times\left[\exp (s \omega W) M(i \omega) \int_{0}^{1} i \exp (-t \omega W) W f(t) d t-\int_{0}^{s} i \exp (-[t-s] \omega W) W f(t) d t\right] d s .
\end{gathered}
$$

Interchanging the orders of integration gives (4.a).

After a bit of manipulation, the resolvent kernel for $t \leq x$, is

$$
\begin{gathered}
R(x, t, \lambda) \\
=\frac{1}{2 \omega} \exp (-x \omega W) M(-i \omega) \exp (2 \omega W)[I-M(i \omega)] \exp (-t \omega W) W \\
+\frac{1}{2 \omega} \exp (-x \omega W)[M(-i \omega)-I] M(i \omega) \exp (-t \omega W) W \\
+\frac{1}{2 \omega} \exp (-x \omega W)[I-M(-i \omega)] \exp (t \omega W) W \\
+\frac{1}{2 \omega} \exp (x \omega W)[M(i \omega)-I] \exp (-t \omega W) W
\end{gathered}
$$

The functions

$$
M( \pm i \omega)=[I-\exp (\mp \omega W) T]^{-1}
$$

have series representations valid as $\omega \rightarrow \infty($ or $\lambda \rightarrow-\infty)$. These are

$$
\begin{gathered}
M(i \omega)=\sum_{k=0}^{\infty}(\exp (-\omega W) T)^{k} \simeq I+\exp (-\omega W) T \\
M(-i \omega)=-T^{-1} \exp (-\omega W) \sum_{k=0}^{\infty}\left(T^{-1} \exp (-\omega W)\right)^{k} \simeq-T^{-1} \exp (-\omega W) .
\end{gathered}
$$

Inserting these series into the expression for the diagonal of the resolvent kernel gives

$$
2 \omega R\left(x, x,-\omega^{2}\right) W^{-1}=J_{1}+\cdots+J_{4}+E(x, \omega)
$$

where the $J_{j}$ use terms up to $k=1$ in the series and $E$ represents the remainder. More explicitly,

$$
J_{1}=\exp (-2 x \omega W), \quad J_{2}=-\exp (-2 x \omega W)+E_{1}(x, \omega)
$$

$J_{3}=I+\exp (-x \omega W) T^{-1} \exp (-[1-x] \omega W), \quad J_{4}=\exp (-[1-x] \omega W) T \exp (-x \omega W)$.

Notice that $J_{4}$, and $J_{3}-I$, and all of their derivatives with respect to $x$ and $\omega$, are $O\left(\exp \left(-\min _{n} w_{n} / 2 \omega\right)\right)$ as $\omega \rightarrow \infty$. The same is true for $E(x, \omega)$ and $E_{1}(x, \omega)$. 
4.2. The trace of $R_{p}(\lambda)$. If $p$ is a bounded real valued function on $\mathcal{G}$, then the operator $-D^{2}-p$ is self adjoint $\left[12\right.$, p. 287] on the domain of $-D^{2}$. Its resolvent will be denoted by

$$
R_{p}(\lambda)=\left[-D^{2}-p-\lambda\right]^{-1}
$$

These resolvents may be represented as $N \times N$ matrices of integral operators,

$$
R_{p}(\lambda) f=\left(\begin{array}{c}
\sum_{m=1}^{N} \int_{0}^{1} R_{m 1}(x, t, \lambda) f_{m}(t) d t \\
\vdots \\
\sum_{m=1}^{N} \int_{0}^{1} R_{m N}(x, t, \lambda) f_{m}(t) d t
\end{array}\right),
$$

where the matrix entries $R_{m n}(x, t, \lambda)$ are continuous functions of $(x, t) \in[0,1] \times[0,1]$ for $\lambda$ in the resolvent set. This result may be developed by mimicking the classical proof $[5$, p. 192] in case $N=1$. An alternative is to first consider the decoupled problem with periodic boundary conditions $f_{n}(0)=f_{n}(1)$. In this case the resolvent may be represented as a diagonal matrix of integral operators, and the change of boundary conditions has a minor effect which may be analyzed as in [12, p. 188].

The distribution of eigenvalues $\mu_{k}$ of $-D^{2}-p$ will look roughly like that of the squares of integers $k^{2}$. Let $\mathcal{N}(\lambda)$ be the eigenvalue counting function

$$
\mathcal{N}(\lambda)=\#\left\{\mu_{k} \leq \lambda\right\} .
$$

The counting function will satisfy the coarse estimate [2, p. 174]

$$
c_{1} \lambda^{1 / 2} \leq \mathcal{N}(\lambda) \leq c_{2} \lambda^{1 / 2}, \quad \lambda>1, \quad c_{1}, c_{2}>0,
$$

which suffices for our needs.

Lemma 4.2. The eigenfunctions $\phi_{k}(x)$ of $-D^{2}-p$ satisfy an estimate

$$
\left|\phi_{k, n}(x)\right| \leq C\left\|\phi_{k}\right\|_{2}, \quad \phi_{k}=\left(\begin{array}{c}
\phi_{k, 1} \\
\vdots \\
\phi_{k, N}
\end{array}\right)
$$

where $C$ is independent of $k$.

Proof. The components of eigenfunctions satisfy the equations

$$
-\phi_{k, n}^{\prime \prime}(x)-w_{n}^{2} p_{n}(x) \phi_{k, n}(x)=w_{n}^{2} \mu_{k} \phi_{k, n}(x), \quad x \in[0,1] .
$$

Each component may be written as a linear combination

$$
\phi_{k, n}(x)=a y_{1}\left(x, \mu_{k}\right)+b y_{2}\left(x, \mu_{k}\right)
$$

where the $y_{j}$ satisfy the same equations and have the initial data

$$
y_{j}^{(i-1)}=\delta_{i j}, \quad i, j=1,2 .
$$

When $p=0$ the solution with the same initial data is

$$
a \cos \left(w_{n} \sqrt{\mu_{k}} x\right)+b \frac{\sin \left(w_{n} \sqrt{\mu_{k}} x\right)}{w_{n} \sqrt{\mu_{k}}}=c \cos \left(w_{n} \sqrt{\mu_{k}} x-\alpha\right),
$$

where

$$
c^{2}=a^{2}+\frac{b^{2}}{w_{n}^{2}\left|\mu_{k}\right|}
$$


Thus

$$
\begin{gathered}
\int_{0}^{1}\left|b \frac{\sin \left(w_{n} \sqrt{\mu_{k}} x\right)}{w_{n} \sqrt{\mu_{k}}}+a \cos \left(w_{n} \sqrt{\mu_{k}} x\right)\right|^{2} d x \\
=\left[a^{2}+\frac{b^{2}}{w_{n}^{2}\left|\mu_{k}\right|}\right]\left[\frac{1}{2}+\frac{1}{4 w_{n} \sqrt{\mu_{k}}} \sin \left(2 w_{n} \sqrt{\mu_{k}} x-\alpha\right)\right] \rightarrow \frac{1}{2}\left[a^{2}+\frac{b^{2}}{w_{n}^{2}\left|\mu_{k}\right|}\right], \quad \mu_{k} \rightarrow \infty .
\end{gathered}
$$

We also have the standard estimates $[16$, p. 13]

$$
\begin{gathered}
\left|y_{1}\left(x, \mu_{k}\right)-\cos \left(w_{n} \sqrt{\mu_{k}} x\right)\right| \leq \frac{C}{w_{n} \sqrt{\mu_{k}}}, \\
\left|y_{2}\left(x, \mu_{k}\right)-\frac{\sin \left(w_{n} \sqrt{\mu_{k}} x\right)}{w_{n} \sqrt{\mu_{k}}}\right| \leq \frac{C}{w_{n}^{2} \mu_{k}},
\end{gathered}
$$

for some constant $C$ independent of $k$ and $\mu_{k} \geq 1$.

If $\|\cdot\|$ denotes the $L^{2}$ norm on $[0,1]$, then for $\mu_{k}$ sufficiently large an application of the triangle inequality yields

$$
\left\|a y_{1}\left(x, \mu_{k}\right)+b y_{2}\left(x, \mu_{k}\right)\right\| \geq \frac{1}{2}\left(a^{2}+\frac{b^{2}}{w_{n}^{2} \mu_{k}}\right)^{1 / 2}-C\left(\frac{|a|}{w_{n} \sqrt{\mu_{k}}}+\frac{|b|}{w_{n}^{2} \mu_{k}}\right) .
$$

This inequality shows that

$$
\max \left(a, \frac{b}{w_{n} \sqrt{\mu_{k}}}\right) \leq C\left\|a y_{1}\left(x, \mu_{k}\right)+b y_{2}\left(x, \mu_{k}\right)\right\| .
$$

Another application of the estimates (4.b) gives the result.

The resolvents and certain other functions of $-D^{2}-p$ will belong to the trace class (see [17, pp. 206-212], [12, pp. 523-525] or [18, pp. 249-260]). For operators $A$ in the trace class, the trace is defined by

$$
\sum_{k}\left(A \phi_{k}, \phi_{k}\right)
$$

for any orthonormal basis $\left\{\phi_{k}\right\}$. The trace norm is $\|A\|_{1}=\operatorname{tr}\left(\left[A^{*} A\right]^{1 / 2}\right)$. The bounds on eigenfunctions of $-D^{2}-p$ can be used to justify a more convenient representation of the trace. This result, presented in the next lemma, is well known in similar contexts $[18$, p. 259].

Lemma 4.3. Suppose that a function $h: \sigma\left(-D^{2}-p\right) \rightarrow \mathcal{C}$ satisfies $\sum_{k}\left|h\left(\mu_{k}\right)\right|<$ $\infty$. The operator

$$
A=h\left(-D^{2}-p\right): \bigoplus_{n} L^{2}\left([0,1], w_{n}\right) \rightarrow \bigoplus_{n} L^{2}\left([0,1], w_{n}\right)
$$

is trace class, and may be represented as a matrix integral operator with kernel $A_{m, n}(x, t)$. The functions $A_{m, n}(x, t)$ may be chosen continuous in $(x, t)$, and with this choice

$$
\operatorname{tr}(A)=\sum_{n=1}^{N} \int_{0}^{1} A_{n, n}(x, x) d x .
$$

Proof. Use an orthonormal basis of eigenfunctions $\phi_{k}$ to write $f \in \bigoplus_{n} L^{2}\left([0,1], w_{n}\right)$ as $f=\sum_{k} a_{k} \phi_{k}$. Since $A f=\sum_{k} a_{k} h\left(\mu_{k}\right) \phi_{k}$,

$$
A f(x)=\int_{0}^{1}\left(\begin{array}{c}
\sum_{n=1}^{N} A_{1, n}(x, t) f_{n}(t) \\
\vdots \\
\sum_{n=1}^{N} A_{N, n}(x, t) f_{n}(t)
\end{array}\right) d t
$$


where the kernel of $A$ has the representation

$$
A(x, t)=\left(A_{m, n}(x, t)\right), \quad A_{m, n}(x, t)=\sum_{k} h\left(\mu_{k}\right) \phi_{k, m}(x) \overline{w_{n} \phi_{k, n}(t)} .
$$

By virtue of our assumptions on $h$ and the bounds on orthonormal eigenfunctions in Lemma 4.2, the series in (4.c) converges uniformly to a continuous function. Note in particular that the coarse eigenvalue estimates for $-D^{2}-p$ imply that the resolvents $R_{p}(\lambda)$ are in this class.

The trace takes the form

$$
\begin{gathered}
\sum_{k}\left(A \phi_{k}, \phi_{k}\right)=\sum_{k} \sum_{n=1}^{N} \int_{0}^{1}\left(A \phi_{k}\right)_{n}(x) \overline{\phi_{k, n}(x)} w_{n} d x \\
=\sum_{k} \sum_{n=1}^{N} \int_{0}^{1} h\left(\mu_{k}\right) \phi_{k, n}(x) \overline{\phi_{k, n}(x)} w_{n} d x=\sum_{n=1}^{N} \int_{0}^{1} A_{n, n}(x, x) d x .
\end{gathered}
$$

To proceed further it will be convenient to assume that derivatives $p^{(j)}$ of the function $p$ are continuous on the graph $\mathcal{G}$. This means that the various components $p_{n}$ of $p$ have $j$ continuous derivatives on $(0,1)$, that these derivatives extend continuously to the closed interval $[0,1]$, and that at the endpoints

$$
\lim _{x \rightarrow 0}\left[\frac{1}{w_{n}} \partial_{x}\right]^{j} p_{n}(x)=\lim _{x \rightarrow 1}\left[\frac{1}{w_{m}} \partial_{x}\right]^{j} p_{m}(x)
$$

for all edges $e_{m}, e_{n}$ respectively entering and exiting a common vertex. Continuity of enough derivatives $p^{(j)}$ on the graph, and particularly across the vertices, will imply that multiplication by $p$ leaves the domain of $-D^{2}$ invariant. This will result in a simple commutation formula (4.j) for $p$ and the resolvent $R(\lambda)$. The commutation formula will lead to an expansion for $R_{p}(\lambda)$ and expressions for the residues of the zeta function $\zeta_{p}(s)$ in which the matrix $T$ does not appear. The symbol $H_{n, k}(p)$ will denote a polynomial in derivatives of $p$.

Lemma 4.4. Suppose that $p^{(j)}$ extends continuously to the graph for $j \leq \mathcal{J}, \mathcal{J} \geq 2$. Then for $\operatorname{Re}(\lambda)<-\|p\|_{\infty}$,

$$
R_{p}(\lambda)=\sum_{n=2}^{\mathcal{J}}\left[\sum_{k=\lceil n / 2\rceil}^{2 n} H_{n, k}(p) D^{2 k-n} R^{k}\right]+E_{\mathcal{J}},
$$

where the error $E_{\mathcal{J}}$ satisfies the estimates

$$
\left\|E_{\mathcal{J}}\right\|=O\left(\omega^{-\mathcal{J}-4}\right), \quad\left\|E_{\mathcal{J}}\right\|_{1}=O\left(\omega^{-\mathcal{J}-3}\right), \quad \omega^{2}=-\lambda .
$$

The polynomials $H_{n, k}(p)$ are the same for all unitary matrices $T(v)$ defining the domain of $i D$.

Proof. Since $p$ is bounded, the perturbation series

$$
R_{p}(\lambda)=R \sum_{n=0}^{\infty}[p R]^{n}=R+R p R+R p R p R+\ldots
$$

will converge if the distance from $\lambda$ to the spectrum of $-D^{2}$ exceeds $\|p\|_{\infty}$.

For the $n$th edge the product rule gives

$$
\left(-\partial_{x}^{2} / w_{n}^{2}-\lambda\right) p_{n} f=p_{n}\left(-\partial_{x}^{2} / w_{n}^{2}-\lambda\right) f-2 \frac{\partial_{x} p_{n}}{w_{n}^{2}} \partial_{x} f-\frac{\partial_{x}^{2} p_{n}}{w_{n}^{2}} f .
$$


If $f=R(\lambda) h$ where $h$ is continuous on each edge, then

$$
\begin{gathered}
\left(-\partial_{x}^{2} / w_{n}^{2}-\lambda\right) p_{n} R(\lambda) h=\left[p_{n}\left(-\partial_{x}^{2} / w_{n}^{2}-\lambda\right)-2 \frac{\partial_{x} p_{n}}{w_{n}^{2}} \partial_{x}-\frac{\partial_{x}^{2} p_{n}}{w_{n}^{2}}\right] R(\lambda) h \\
=p_{n} h-\left[2 \frac{\partial_{x} p_{n}}{w_{n}^{2}} \partial_{x}+\frac{\partial_{x}^{2} p_{n}}{w_{n}^{2}}\right] R(\lambda) h .
\end{gathered}
$$

This equation extends by continuity to all $h \in L^{2}(\mathcal{G})$.

If $p^{(j)}, j=0,1,2$, extend continuously across the vertices, multiplication by $p$ maps the domain of $-D^{2}$ into itself, so that

$$
R(\lambda) p_{n}=p_{n} R(\lambda)+2 R(\lambda) \frac{\partial_{x} p_{n}}{w_{n}^{2}} \partial_{x} R(\lambda)+R(\lambda) \frac{\partial_{x}^{2} p_{n}}{w_{n}^{2}} R(\lambda) .
$$

Interpreting these equations globally on the graph, we will write

$$
R(\lambda) p=p R(\lambda)+2 R(\lambda) p^{\prime} D R(\lambda)+R(\lambda) p^{\prime \prime} R(\lambda), \quad p^{\prime}=D p .
$$

Now start with the identity

$$
R_{p}(\lambda)=R\left[\sum_{n=0}^{\mathcal{J}-1}[p R]^{n}+(p R)^{\mathcal{J}}(1-p R)^{-1}\right] .
$$

Since multiplication by $p$ leaves the domain of $\left[-D^{2}\right]^{k}$ invariant, the term

$$
R(p R)^{\mathcal{J}}(1-p R)^{-1}
$$

maps $L^{2}(\mathcal{G})$ into the domain of $\left[-D^{2}\right]^{\mathcal{J}+1}$ and satisfies the norm estimate

$$
\left\|R(p R)^{\mathcal{J}}(1-p R)^{-1}\right\| \leq K_{\mathcal{J}}|\lambda|^{-\mathcal{J}-1}, \quad \lambda<0 .
$$

Thanks to the trace norm estimate $([17$, p. 218$]$ or $[18$, p. 256])

$$
\|A B\|_{1} \leq\|A\|_{1}\|B\|
$$

and Lemmas 4.1 and 4.3 ,

$$
\left\|R(p R)^{\mathcal{J}}(1-p R)^{-1}\right\|_{1} \leq K_{\mathcal{J}}|\lambda|^{-\mathcal{J}}\|R\|_{1} \leq K_{\mathcal{J}}|\lambda|^{-\mathcal{J}-1 / 2}, \quad \lambda<0 .
$$

Note that an operator of the form $D^{j} R^{k}$ with $j+1<2 k$ will map $L^{2}(\mathcal{G})$ into the domain of $D^{2 k-j}$ with the norm estimate

$$
\left\|D^{j} R^{k}\right\| \leq \omega^{2 k-j}, \quad \omega^{2}=-\lambda>0,
$$

and the trace norm estimate

$$
\left\|D^{j} R^{k}\right\|_{1} \leq \omega^{2 k-j-1} .
$$

Thus the commutation formula (4.d) allows us to rewrite $R \sum_{n=0}^{\mathcal{J}-1}[p R]^{n}$ by pushing powers of $R$ to the right until the desired form,

$$
R_{p}(\lambda)=\sum_{n=2}^{\mathcal{J}}\left[\sum_{k=\lceil n / 2\rceil}^{2 n} H_{n, k}(p) D^{2 k-n} R^{k}\right]+E_{\mathcal{J}},
$$

and error estimates are achieved. Finally, the unitary matrices $T(v)$ defining the domain of $i D$ did not enter into the generation of the polynomials $H_{n, k}(p)$. 
4.3. The zeta function for $-D^{2}-p$. Having established Lemmas 4.1, 4.3, and 4.4 , it is now possible to obtain a description of the zeta function $\zeta_{p}(s)$ associated to a Schrödinger operator $L=-D^{2}-p$ on a graph $\mathcal{G}$. For completeness we briefly sketch the development. A systematic treatment for the case of a manifold, which is quite similar, may be found in [18, pp. $82-114]$.

On one hand, $\zeta_{p}(s)$ has the simple description

$$
\zeta_{p}(s)=\sum_{k} \mu_{k}^{s}, \quad R e(s)<-1 / 2,
$$

where $\left\{\mu_{k}\right\}$ is the sequence of (nonzero) eigenvalues of $L$, taken with multiplicity. This function is the same as $\operatorname{tr}\left(L^{s}\right)$, which has an alternate contour integral description.

Theorem 4.5. Suppose that $p^{(i)}$ extends continuously to the graph for $i \leq \mathcal{J}$, $\mathcal{J} \geq 2$. The function $\zeta_{p}(s)$ has a meromorphic extension to $\operatorname{Re}(s)<(\mathcal{J}+1) / 2$, with poles located at the points $s=(2 j-1) / 2$ for $j=0,1,2, \ldots$ The poles are all simple, and the residues have the form

$$
\sum_{n=1}^{\mathcal{J}} \int_{0}^{1} \mathcal{H}_{j, n}\left(p_{n}\right) d x
$$

where $\mathcal{H}_{j, n}(p)$ is a polynomial in $p_{n}$ and its derivatives. The residues are independent of the particular choice of unitary matrices $T(v): \mathcal{C}^{\delta(v)} \rightarrow \mathcal{C}^{\delta(v)}$.

Sketch of Proof. Slit the complex plane along the negative real axis, so that $\log (\lambda)$ is analytic in the complement of the slit, and consider the contour $\Gamma=\Gamma_{1} \cup \Gamma_{2} \cup \Gamma_{3}$ which contains the slit, where

$$
\Gamma_{1}=t e^{i \pi}, \quad \infty>t>r, \quad \Gamma_{3}=t e^{-i \pi}, \quad r<t<\infty, \quad \Gamma_{2}=r e^{-i \theta}, \quad-\pi \leq \theta \leq \pi .
$$

If all eigenvalues of $L$ are greater than $r$, then (taking the logarithm real for $\lambda>0)$

$$
L^{s}=\frac{1}{2 \pi i} \int_{\Gamma} \lambda^{s} R(\lambda) d \lambda=\frac{1}{2 \pi i} \int_{\Gamma} e^{s \log (\lambda)} R(\lambda) d \lambda, \quad \operatorname{Re}(s)<0,
$$

and

$$
\operatorname{tr}\left(L^{s}\right)=\frac{1}{2 \pi i} \int_{\Gamma} \lambda^{s} \operatorname{tr}(R(\lambda)) d \lambda .
$$

If $L$ has nonpositive eigenvalues, choose $r$ so that $\Gamma_{2}$ lies in the resolvent set of $L$ and encloses the nonpositive eigenvalues. Then the contour integral (4.e) will give

$$
\sum_{\mu_{k}>r} \mu_{k}^{s}
$$

which differs from $\zeta_{p}(s)$ by an entire function.

The expansion of $R_{p}(\lambda)$ from Lemma 4.4. is now inserted into (4.e). The estimate $\left\|E_{\mathcal{J}}\right\|_{1}=O\left(\omega^{-\mathcal{J}-3}\right)$ implies that

$$
\frac{1}{2 \pi i} \int_{\Gamma} \lambda^{s} \operatorname{tr}\left(E_{\mathcal{J}}\right) d \lambda
$$

defines an analytic function of $s$ for $\operatorname{Re}(s)<(\mathcal{J}+1) / 2$. The remaining terms have the form

$$
\frac{1}{2 \pi i} \int_{\Gamma} \lambda^{s} \operatorname{tr}\left(H_{n, k}(p) D^{2 k-n} R^{k}\right) d \lambda
$$


The powers of the resolvent satisfy

$$
R(\lambda)^{j+1}=\frac{1}{2 \pi i} \frac{1}{j !} \partial_{\lambda}^{j} R(\lambda),
$$

so that by Lemma 4.1 the only terms from (4.f) contributing to the singularities of $\zeta_{p}(s)$ are those with no positive powers of $D$.

By Lemmas 4.1 and 4.3 the significant terms from (4.f) may be rewritten as

$$
\left[\sum_{n} \int_{0}^{1} w_{n} H_{n, n / 2}(p) d x\right] \frac{1}{2 \pi i} \int_{\Gamma} \lambda^{s} \frac{1}{(k-1) !} \partial_{\lambda}^{k-1}(2 \omega)^{-1} d \lambda
$$

One calculates that the singular part of the contour integral is

$$
\frac{1 \cdot 3 \cdots(2 k-3)}{2^{k}(k-1) !} \frac{\sin (\pi s)}{\pi} \frac{-1}{s-[2 k-3] / 2}, \quad s<[2 k-3] / 2 .
$$

This establishes the existence of a meromorphic continuation for $\zeta_{p}(s)$, and the description of the singular part.

Finally, notice that the particular unitary vertex matrices $T(v)$ played no role in the description of the singular part of $\zeta_{p}(s)$.

One may use the method of proof to calculate the residues, although the computations quickly become tedious. The first few residues are recorded in the following table.

$$
\begin{aligned}
& \underline{\text { pole }} \underline{\text { residue }} \\
& s=-1 / 2 \quad \sum_{n} w_{n} /[2 \pi] \\
& s=1 / 2 \quad-\left[\sum_{n}\left(\int_{0}^{1} w_{n} p_{n}\right)\right] /[4 \pi] \quad . \\
& s=3 / 2 \quad 3\left[\sum_{n}\left(\int_{0}^{1} w_{n} p_{n}^{2}\right)\right] /[16 \pi] \\
& s=5 / 2 \quad-5\left[\sum_{n}\left(\int_{0}^{1} w_{n}\left[p_{n}^{3}-(1 / 2)\left(\partial_{x} p_{n} / w_{n}\right)^{2}\right]\right)\right] /[32 \pi]
\end{aligned}
$$

A simpler approach is to make a judicious choice of the matrices $T(v)$. Partition $\mathcal{G}$ into edge disjoint loops with no repeated edges, as in Proposition 3.2. If $v$ is a vertex in one of the loops, with input edge $i$ and output edge $j$, let $T$ be defined by requiring $f_{i}(1)=f_{j}(0)$. In this case after a change of coordinates the operator $-D^{2}-p$ may be recognized as a diagonal operator

$$
\frac{1}{2 \pi}\left(\begin{array}{cccc}
-\partial_{s}^{2}-\tilde{p}_{1} & 0 & \ldots & 0 \\
0 & -\partial_{s}^{2}-\tilde{p}_{2} & \ldots & 0 \\
0 & 0 & \ldots & -\partial_{s}^{2}-\tilde{p}_{M}
\end{array}\right)
$$

acting on $\bigoplus_{m} L^{2}\left(S_{r(m)}^{1}\right)$, where $S_{r(m)}^{1}$ denotes the circle of radius $r(m)$ and $s$ is arc length. Thus residue computations may take advantage of the previously developed art $[8,14]$.

Our main interest in the development of $\zeta_{p}(s)$ was to see that the set of operators $-D^{2}-p$ having the same eigenvalues is compact. The argument is familiar. If the eigenvalues are given, then $\zeta_{p}(s)$ is determined, and in particular the residues are fixed. One shows from the explicit form of the residues that both $p$ and its derivative are bounded in $L^{2}$. The details of a more general version of this argument may be found in $[14$, p. 226], where the equivalent heat kernel invariants $[11$, p. 56], [18, pp. 113-114] are used. Thus the following result is obtained. 
Corollary 4.6. Consider potentials $p, q$ with at least 5 continuous derivatives on $\mathcal{G}$. The isospectral sets

$$
M_{p}=\left\{q \mid \mu_{k}(q)=\mu_{k}(p), \quad k=1,2,3, \ldots\right\}
$$

are compact in $\bigoplus_{n} L^{2}\left([0,1], w_{n}\right)$.

\section{REFERENCES}

1. L. Ahlfors, Complex analysis, McGraw-Hill, New York, 1966. MR 32:5844

2. R. Carlson, Expansions associated with non-self-adjoint boundary-value problems, Proceedings of the American Mathematical Society 73 (1979), no. 2, 173-179. MR 80e:47040

3. F. Chung, Spectral graph theory, American Mathematical Society, 1997. MR 97k:58183

4. F. Chung and S. Sternberg, Laplacian and vibrational spectra for homogeneous graphs, Journal of Graph Theory 16 (1992), no. 6, 605-627. MR 93j:58135

5. E.A. Coddington and N. Levinson, Theory of ordinary differential equations, McGraw-Hill, New York, 1955. MR 16:1022b

6. E. Davies, Large deviations for heat kernels on graphs, Journal of the London Mathematical Society (2) 47 (1993), 65-72. MR 94f:58135

7. Y. Colin de Verdiere, Spectre du Laplacien et longueurs des geodesiques periodiques II, Compositio Mathematica 27 (1973), no. 2, 159-184. MR 50:1293

8. L. Dikii, Trace formulas for Sturm-Liouville operators, American Mathematical Society Translations 18 (1958), 81-115. MR 23:A1874

9. P. Exner and P. Seba, Schroedinger operators on unusual manifolds, Ideas and methods in quantum and statistical physics (Oslo 1988) (S. Albeverio, J. Fenstad, H. Holden, and T. Lindstrom, eds.), 1992, pp. 227-253. MR 94a:81026

10. N. Gerasimenko and B. Pavlov, Scattering problems on noncompact graphs, Theoretical and Mathematical Physics 74 (1988), no. 3, 230-240. MR 90f:47010

11. P. Gilkey, The index theorem and the heat equation, Publish or Perish, Boston, 1974. MR 56:16704

12. T. Kato, Perturbation theory for linear operators, Springer, New York, 1995. MR 96a:47025

13. B.M. Levitan and V.V. Zhikov, Almost periodic functions and differential equations, Cambridge University Press, Cambridge, 1982. MR 84g:34004

14. H. McKean and P. van Moerbeke, The spectrum of Hill's equation, Inventiones Math. 30 (1975), 217-274. MR 53:936

15. B. Mohar and W. Woess, A survey on spectra of infinite graphs, Bull. London Math. Soc. 21 (1989), 209-234. MR 90d:05162

16. J. Poschel and E. Trubowitz, Inverse spectral theory, Academic Press, Orlando, 1987. MR 89b:34061

17. M. Reed and B. Simon, Methods of modern mathematical physics, 1, Academic Press, New York, 1972. MR 58:12429a

18. M. Shubin, Pseudodifferential operators and spectral theory, Springer-Verlag, Berlin, 1987. MR 88c:47105

19. P. Sy and T. Sunada, Discrete Schrödinger operators on a graph, Nagoya Math Journal 125 (1992), 141-150. MR 93c:58232

Department of Mathematics, University of Colorado at Colorado Springs, ColORADO Springs, Colorado 80933

E-mail address: carlson@vision.uccs.edu 